\title{
An open system model of ecological architecture
}

\author{
Y. Gu \& R. Evans \\ Victoria Research Laboratory, National Information and Communication \\ Technology Australia, Department of Electrical and Electronic \\ Engineering, University of Melbourne, Australia
}

\begin{abstract}
This paper presents a model of open systems evolution as a paradigm for ecological design in architecture. Based on evolutionary thermodynamics and complex systems science, the model of open systems evolution is constituted by the mechanisms of the adaptation of open systems to the host environment via natural gradients, the self-organization of open systems so as to optimize resource distributions according to the maximum entropy principle, the generation of diversity and the production of minimal entropy in the host environment. According to this model, the authors propose a conceptual framework for ecological architecture that describes the ecological interactions of buildings with the natural environment in open thermodynamic terms, and actively engages the end-users in buildings into the micro-climate control. In the manner of open systems evolution, these multiple interactions evolve to optimize the environmental performance of buildings, resulting in a sustainable symbiosis of architecture and nature.
\end{abstract}

Keywords: open systems evolution, ecological architecture, intelligent design.

\section{Introduction}

Induced by human activities, including industrial development and production, urbanisation and agriculture over the last two centuries, global warming and climate change are recognised as an entropic consequence of environmental depletion (Ingersoll [7]; Moore [13]). The environmental crisis has been and will continue to be a serious threat to basic social well-being and global stability, presenting the world with a major dilemma and challenge of achieving 
sustainable development in the $21^{\text {st }}$ century. On such a background, a conceptual systems theory framework for sustainable development is described in this paper.

Crisis is often a prelude to the emergence of new theories, and philosophical thinking is a powerful device to unlock riddles in any field (Kuhn [11]). The environmental crisis caused by industrial development raises the urgent need to explore a new theoretical framework for development. Based on the theory of evolutionary thermodynamics (Prigogine [15]) and complex systems science (Kauffman [9], [5]), in this paper the authors generalise the mechanisms of open systems evolution and introduce a new paradigm for ecological architecture and sustainable design in the built environment.

\section{Open system evolution}

In thermodynamics an open system refers to a system that is able to make use of available resources from the host environment for its evolution, driven by naturally arising pressure, density, temperature gradients etc, between the system and the host environment. At the macroscopic level, an open system evolves from an initial chaotic state to a non-equilibrium state or a steady state when order emerges in the open system, which is indicated by a zero entropy rate and a minimal value of entropy. Subsequently the open system will maintain this stable interrelation with its host environment. In other words the open system is compatible with the constraints of the host environment.

At the microscopic level an open system exhibits a mechanism of selforganisation during its evolutionary phase whereby an organised structure gradually emerges such that the distribution of resources in the system is optimized according to the maximum entropy principle. Corresponding to this microscopic level self-organisation, at the macroscopic level there is reduction in entropy transfer by the open system to its host environment. This final state, composed of the emergence of a highly ordered structure for an optimal distribution of input resources in the system and the minimised entropy produced by the system, is identified as the emergence of order in the open system.

In abstract terms, the evolution of an open system can be thought of as the open system adapting to the host environment spontaneously due to natural gradients. During the evolution, indicated by entropy, the system is aware of its on-going performance, aware of its negative impact upon the host environment. The system adapts to the host environment by self-organising a highly organised internal structure for resource distribution aimed at minimising entropy thus optimising the system's interrelation with the host environment. These desirable consequences, i.e. the ordered structure, minimised entropy and stable interactions, are identified as the order of the open system after evolution.

To interpret the black-box description of open systems evolution, we assume, it is the internal structure being self-organised by the open system which reduces the rate of entropy towards zero and minimises the value of entropy at the arrival of a stable state. In other words, we assume that the entropy jointly produced by the inputs from the host environment at the macroscopic level and by the selforganisation of the open system at the microscopic level fully describes 
self-organisation of an open system during the evolution towards an ordered state. The following section specifies the mechanism of self-organisation in more detail.

\subsection{Mechanisms of self-organisation}

At the local level in response to the global evolution of an open system, selforganisation without central power (Prokopenko et al. [17]) is a primary mechanism to process the evolution dynamics within the system in response to stimuli from the host environment, e.g. inputs of energy, matter and information. The term, self-organisation, has deep roots in biological science, related to the origin of life (Kauffman [10]), e.g. chemical bonding at molecular level. It is related to resource allocation within a system, resulting in an increase in complexity (Adami [1]; Prokopenko et al. [17]).

During the evolution of an open system, after entering into the system across the boundary, the input resources will be released within the system in an evolutionary manner that finishes in a non-equilibrium state of the open system when entropy is minimised. Starting from the release of the input resources, the open system self-organises itself in the following steps:

i) A structure is self-generated internally by the system after the release of the input resources, which function to control, channel and distribute the resources, and also constrain and limit the distribution;

ii) As a direct result of the structure generation, the input resource are distributed by the structure within the system, and constrained by and subjected to the structure;

iii) Most of the input resources are effectively distributed within the system for useful work by the structure, but some of them are limited and constrained; thus it requires further development of the structure in order to support the ongoing effective release and distribution of resources in the open system;

iv) Gradually, the release and distribution of input resources shape a complex topology emerging in the open system.

In brief, a structure is spontaneously generated by the open system after inputs flowing into the open system are released. It is used for a possibly broadest distribution of inputs resources within the open system. In turn, it produces a complex topology of resource distribution in the system.

Over this complex process of self-organisation, entropy is inevitably transferred from the open system to the host environment. The change of the rate of entropy and the value of entropy, e.g. the reduction or the increase of entropy, indicate the on-going process of self-organisation at the microscopic level in response to the on-going process of evolution of the open system at the macroscopic level. The value of entropy is finally stabilized at a minimal value that indicates the completion of the evolution phase and the emergence of order in the open system. The repetitions of generations of structure for the effective distribution of resources are processed in the direction of reducing the rate of entropy towards zero and minimising the value of entropy till completion of the evolution. 
In summary, the black-box dynamics of self-organisation are composed of the self-generation of structure in the direction of an optimisation of the distribution of inputs resources at a microscopic level. When the evolution of the open system at the macroscopic level is completed, which is indicated by the minimised entropy, complexity and diversity emerge in the system, i.e. a complex topology of resource distribution. Such a complex process of an open system is generalized as the mechanism of self-organisation.

\subsection{Mechanisms of evolution}

The mechanism of self-organisation of an open system at the microscopic level to complete the evolution of the system at the macroscopic level suggests an evolutionary mechanism for optimising resource distribution in a system with minimal negative impact, i.e. entropy produced by an open system to the host environment. Three distinctive transitional phases occur during the evolution of an open system (Prigogine [15]), as shown in Figure 1. Far from the equilibrium state of the system and near the equilibrium state of the system, when the system is growing from being uneven and frustrated with its host environment, and finally a non-equilibrium state of the system when the system is growing so as to be compatible with the host environment.

In the end, the system may gain autonomy from its host environment. If there are some new gradients between the system and the host environment, the open system may start another new round of evolution to develop its complexity. One of economists (Dmitry [3]) argues that it takes 50 years for the global economic system to self-organise and settle-down in a new stable state that lasts only 20 years. This is a good example of an open system evolving from a chaotic state to an ordered state.

During evolution, it is the uncertainty, entropy of a system, which drives the successive evolution of the open system from the far from equilibrium state to the final ordered state, in a feedback-control loop. From this perspective, entropy, or the uncertainty in a system, is the attractor of evolutionary dynamics, without which the evolution and self-organisation cannot take place. When we have an entropic environmental crisis caused by industrial development, it indicates the need for further evolution to minimise entropy as much as possible, for example, by making full use of available resources, e.g. solar, wind, geothermal and some other renewable sources, to replace the use of fossil fuel and to minimise the negative impact, supported by innovative technologies. As physicist Fredrik Keffer argued in 1960 (Rogers [18]), "the early industrial revolution involved energy, but the automatic factory of the future is an entropy revolution. The future belongs to those who can control entropy."

Over the voyage of open systems evolution, there are two arrows of time. The first arrow of time is the irreversible production of entropy by the open system towards the host environment, which is macroscopically observable (Prigogine [16]). The second arrow of time is the emergence of self-organising structure towards an ordered state of the open system at the microscopic level. This results in the complexity and diversity in the open system. In the case of the earth which 


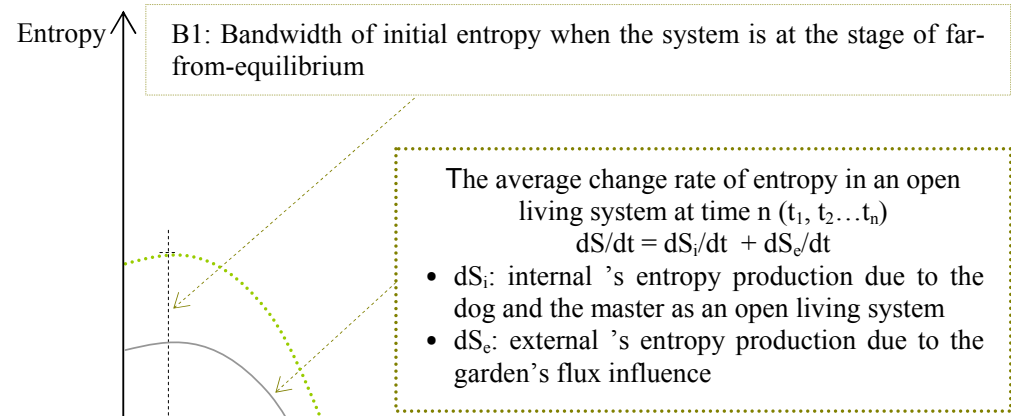

B2: Bandwidth of intermediate entropy when the system is at the stage of near-equilibrium

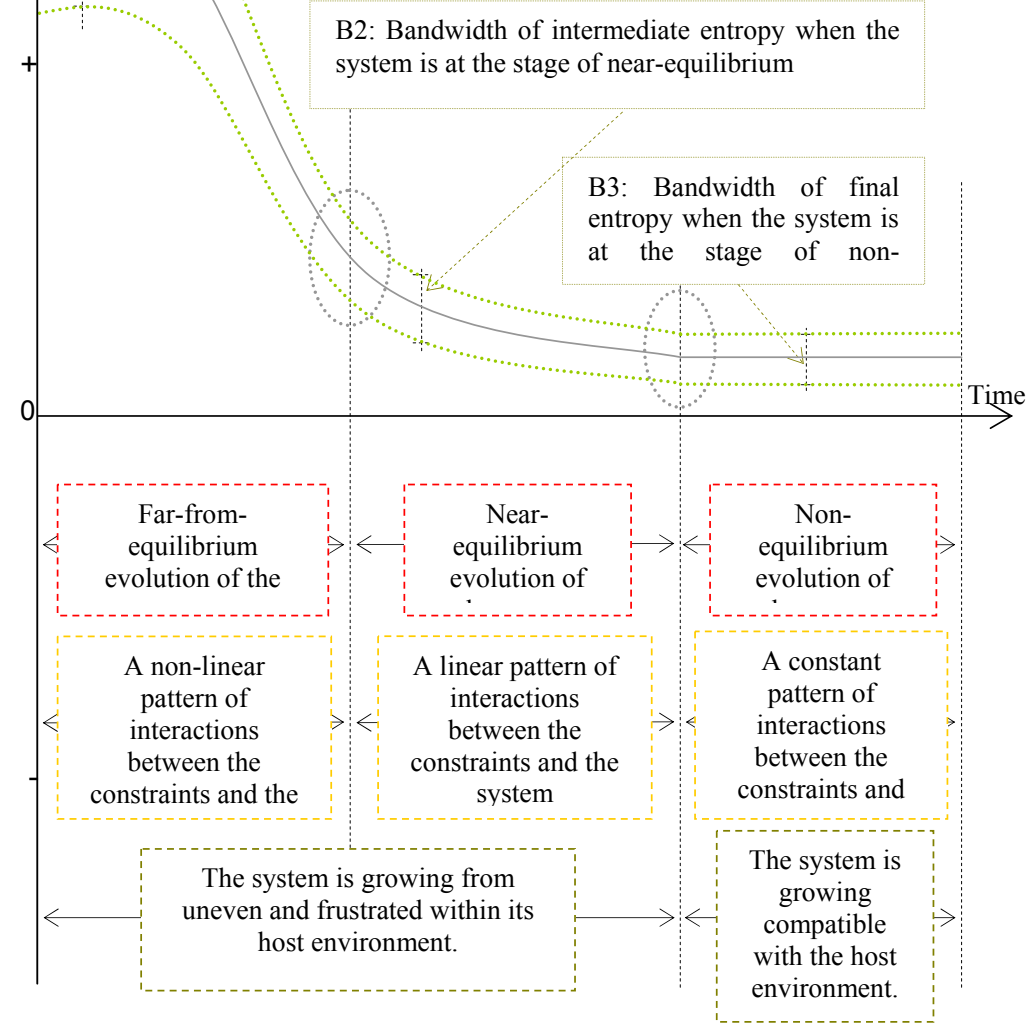

Figure 1: Three transitional phases of an open system evolution.

thermodynamically exchanges energy, e.g. solar energy, with the universe as the host environment, entropy increases irreversibly into the universe; in the meantime, life on the earth is growing to high levels of organisation, complexity and diversity through a steady growth of structure (Davies [4]).

As argued in the previous section, the change of the rate of entropy and the value of entropy indicate an on-going process of evolution. Over the evolution, the production of entropy from the open system into the host environment 
continues accumulating, although it is minimised when evolution completes. This interpretation of open systems evolution is consistent with the classical statement of the Second Law of Thermodynamics which states that the irreversibility of entropy produced by the system, e.g. closed or open systems, into the host environment during a thermodynamic exchange, results in a heat death of the host environment, e.g. the universe as a host environment to the earth as an open system. The difference between an open system and a closed system is the possibility of open systems producing less and less entropy into the host environment due to the continuous inputs supported by the host environment for the successive evolution of the system. However, a closed system does not have the chance of continuous energy and matter input into the system, to reduce the value of entropy produced to the host environment.

In summary, it is by the structure self-generated by the open system in response to the stimulation of input resources from the host environment that an open system is able to complete evolution and realise a compatible interrelation between the system and the host environment. During the dynamic process of self-organisation, the system's performance is dynamically changing as displayed in Figure 1, where the bandwidth and the amplitude of the value of entropy is actually determined by the present structure in the system and the state of the surroundings i.e. the host environment. A desirable structure is a structure which can maximise the distribution of all available resources in the system, committed to all components within the system for minimal entropy. The authors assume this distribution is in accord with the maximum entropy principle as the optimal resource distribution companied with minimised entropy to complete the evolution of the open system. In other words, the more entropy, which is uncertainty of information in a probability sense, the more diversity will be generated by the system. The consequence of self-organisation, i.e. the emergence of complexity and diversity in the system, reinforces the vitality of the system, e.g. increasing the possibility of the further evolution of the system due to the existence of gradients between the agents of the system. This complexity and diversity thus facilitate an efficient transfer of the system from the past to the future, which can be identified as an alterative paradigm for sustainable development and human survival on earth.

\section{Open system evolution in ecological architecture}

The paradigm of open systems evolution is a guiding principle in Nature that seems specifically designed for sustainability. Learning from Nature, several implications of open system evolutions in architecture are generalised as follows.

\subsection{A model of open thermodynamic systems for ecological architecture}

As stated previous, the basic meaning of entropy in thermodynamics theory refers to unavailable and wasted energy during the thermodynamic transformation in a closed system, a dysfunction of the system. Some extensional meanings of entropy in the real world include chaos, disorder, poverty, 
frustration, irreversibility (Prigogine [16]), uncertainty (Shannon [19]) in information science. In contrast, order refers to all the desirable properties, including organisation, intelligence, efficiency, beauty, humanity, and so on. In particular, 'humanity' has been used as a slogan by many politicians to campaign for order in the world. Accordingly, applied to architecture, the authors argue that entropy refers to all the negative environmental impacts of buildings over their life-cycles, and order refers to the optimal environmental performance of buildings, e.g. positive ecological impacts.

A model of evolutionary architecture was initially suggested by Frazer (Frazer [6]) for an alternative to ecological sustainability, which exhibits the metabolism of buildings in such a way that architecture enjoys a thermodynamically open relationship with the environment in both a metabolic and a socio-economic sense. It will maintain its stability with the environment by negative feedback interactions and promote evolution in its employment of positive feedback. It will conserve information while using the processes of autopoiesis, autocatalysis and emergent behaviour to generate new forms and structures. It will be involved with re-adjusting points of disjuncture in the socioeconomic system by operation of positive feedback. It is not a static picture of being, but a dynamic picture of becoming and unfolding, a direct analogy with a description of the natural world.

The authors take this model of open systems further and argue the implication of an open systems model lies in an evolutionary mechanism of open systems for the optimisation of energy and resources consumption in buildings over their life-cycles, to improve the environmental performance of buildings in the context of the natural environment. By efficiently using energy and material fluxes available in the natural environment, it is possible to realise an ecological sustainability, i.e. minimised negative environmental impact towards an ecological compatibility between building systems and their natural ecosystems. The implications of open systems evolution are further specified below.

\subsection{Sustainability through evolutionary optimization}

'Intelligent design' was anticipated by Darwinism. The exquisite, complex structure in nature could have evolved by accumulated random mutations and by natural selection. With the framework of open systems evolution, intelligence means the system able to adapt to the host environment and to use inputs for evolution, aware of its on-going performance and its impact upon the host environment, able to self-organise a highly ordered structure for resource distribution in order to optimise its performance with minimal negative impact, and consequently to generate a compatible interrelation with its host environment, thus achieving long term ecological sustainability.

Within this framework, the concept of sustainability is generalised to attain a balanced ecological interrelation between the system and its natural environment through an evolutionary process. Sustainable design as an alternative design standard in the coming decade is necessary not only to design for energy and resource efficiency, and pollution reduction, but also to design with full responsibility to all the imperatives in a social-economic-environmental context, 
and to design with an awareness of impact in that context, i.e. the natural environment, human beings etc.

In summary, sustainable design should be treated as intelligent design. Hence, the questions raised from the framework of open systems evolution to architecture design are:

i) What is the aim of design? Design for optimal performance of a system, such as a building. The optimisation can be defined as a desirable property in each particular context, e.g. optimised environmental performance in the context of the natural ecosystem;

ii) Which evolutionary phase best describes a system of buildings, the chaotic stage of dynamic evolution or the ordered stage of stable nonequilibrium?

iii) What is the performance of a system of buildings and/or cities? Suppose the performance of the system is determined by the structure and the context, i.e. the system's performance at a macroscopic level is determined by the internal self-organised structure of the system at the microscopic level, and the state in the particular context.

iv) How can we evaluate the performance of the system? Indicated by some referential entropy.

v) Does the system need further evolution? Is the system fully evolved, under-evolved, or un-evolved in the direction of reducing the entropy of the system, i.e. the dysfunction of environmental performance;

vi) Which system and structure are more adaptive and viable for achieving desirable long term ecologically sustainability performance of a building? and

vii) How can we achieve optimisation of system performance using the mechanisms of open systems evolution?

A recent example involving the application of intelligent mechanisms for optimisation is the Taipei Performing Arts Centre, designed by Kokkugai in 2008. Swarm intelligence (Leach [12]) is the emergent properties of this swarm intelligence system, a population composed of a large number of smaller discrete elements, e.g. colonies of ants, flocks of birds, networks of neurons or even the global economy. It displays a bottom-up collective intelligence, generating an active networked topology in which agents self-organise in reforming their topology enabling a gradient interaction between explicit design and emergent processes. The creation of a fresh and highly innovative vocabulary of architectural forms is generated by the algorithmic potential of the computer, including the adaptive, parametric behaviour of distributive systems mutating across a filed condition.

In order to optimize the performance of a building, it is necessary to establish a relevant internal structure that responds to the desirable external performance. For example, by organising a topological structure of energy and resource consumption in response to the optimal environmental performance of a building over its life-cycle, a range of minimised negative environmental impacts can be achieved. This topological structure does not necessarily mean a hierarchical one, but some other alternative forms, e.g. democratic, as suggested by complex 
systems science. It is highly self-organised with the aim of minimising entropy, i.e. negative environmental impact of buildings to the natural environment, to realise ecologically sustainable compatibility of buildings with the natural environment.

In summary, the second implication of open systems evolution to architecture is as an intelligent mechanism for ecological design towards an optimisation of sustainability.

\subsection{A holistic scheme for systematic sustainability in ecological architecture}

Based on the mechanism of self-organization of open systems evolution, the authors propose a holistic scheme for sustainable design with the intelligent mechanism of open systems evolution, as shown in Figure 2. This embraces not only buildings, and the natural environment, but also brings an active participation of the end-users of buildings. In this model, an interactive design scheme for system sustainability is integrated into a holistic information environment, which is open to the participation of end users, concerning the environmental impacts of design and development, a positive engagement of man, nature and buildings all aligned towards sustainability. In other words, through the medium of buildings as man-made intervention into nature, an ecologically positive relationship of man and nature can be achieved.

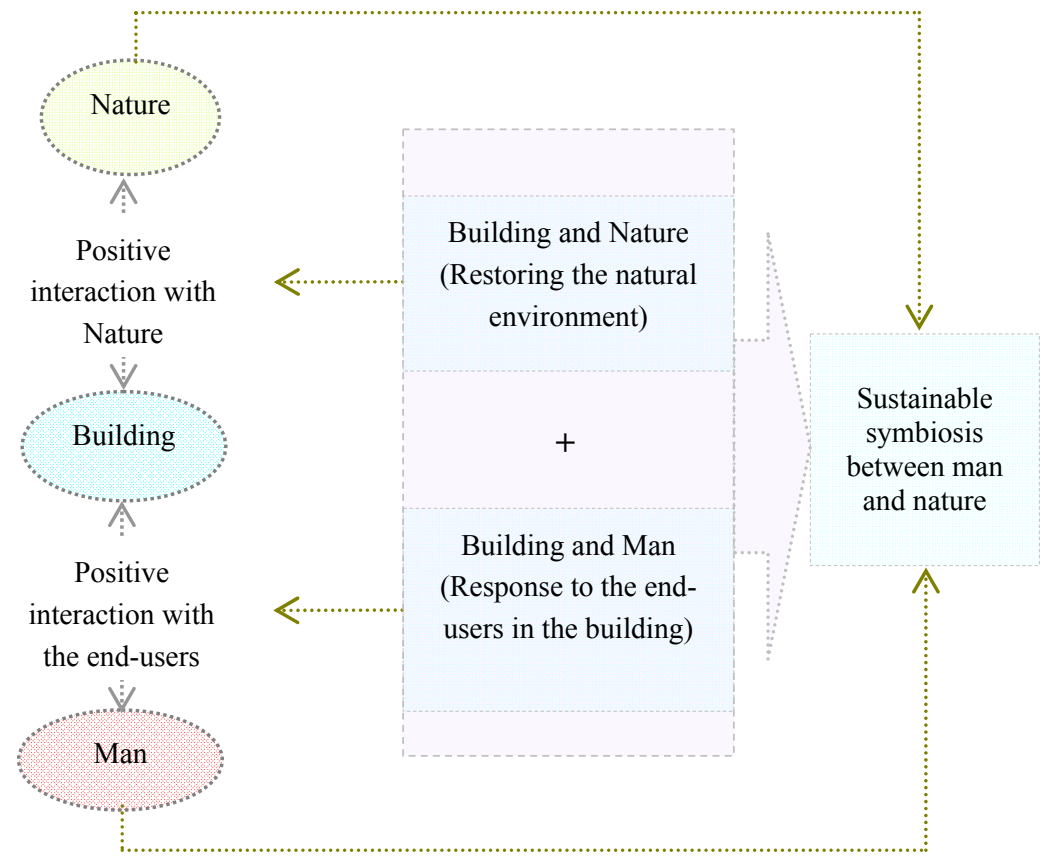

Figure 2: $\quad$ A holistic design scheme for system sustainability in architecture. 
The quest for environmental values in architecture and for a harmonious balance between man and the natural surroundings is not new. In China, "the harmony between the heaven and the human" or "man and nature in one" (Jin [8]) was originally argued by Zhuang-zi, and later developed by Dong Zhong-shu in the Han Dynasty, 200-300 B.C., when the fundamental cultural personality of China were established. In modern times, Jin Yue-lin generalised it as the philosophical foundation of Chinese civilisation over thousands of years. It reflects the agricultural civilization, when tangible knowledge was not fully enough developed to strengthen people with the idea of conquering nature. On the contrary, the ideological dependence on the natural environment was well developed, and provided people with the idea of nature being more powerful than man. Ironically, in our current industrial age, this human dependence on nature is still developing. It implies, compared to the greatness of Nature, how powerless human beings are after more than two hundred years' aggressive and desperate hunting, fighting, and exploiting in the industrial pattern.

\section{Ecological design from concept to creation}

Based on the arguments above, the authors articulate sustainable design at the abstract level as a synthesis of art and science. As is usually the case however the devil is in the detail. Below we begin to unpack some of this detail by sketching two important aspects of our on-going research program.

\subsection{Fluctuation to equilibrium via evolutionary algorithms}

The first sub-project of 'ecological design from concept to the creation' is to simulate the self-organisation of open systems evolution. As argued by (Nicolis and Prigogine [14]) open systems are actually dissipative structures that continuously make use of the inputs for their long-term survival. The attraction of dissipative structures is the transformation between complexity and simplification, between vibrant fluctuation and smooth equilibrium. Certainly, for dissipative structures producing fluctuation is unpleasant and energy consuming. Our work is aimed at developing an approach to speed-up the timeconsuming process of evolution in open systems by efficiently controlling the release of energy so as to achieve sustainable design in the context of architecture.

\subsection{System structure and performance}

The second sub-project is entitled 'the channel of the system performance and the system structure for resources distribution'. We are investigating how the allocation of each individual agent in the system, e.g. the privilege and available access to the resources, the authority of freedom, the scope of the activity field, etc, affects the performance of the system at the global coordination scale; and how system performance is determined by the structure and pattern of resource distribution in the system. 
Until each agent is sufficiently intelligent as indicated by the entropy level (i.e. has evolved enough), the whole system will not work properly as an intelligent system for the optimisation of its performance. The identification of a system's 'intelligence' requires information about the system, the level of uncertainty about the system state etc. For example, as usually suggested from natural evolution theory, each biological system barely develops an adaptive structure to solve the present problem imposed from their niche in Nature.

\section{Conclusion}

In conclusion, self-organisation during the evolutionary phase of an open system is suggested as a mechanism to self-generate a structure for the optimisation of resources distribution. Also self-organisation results in the minimisation of entropy towards the completion of the evolutionary phase. These two desirable effects are identified as the emergence of order in the system. The mechanisms of open systems evolution, generalised in this paper, suggest a new paradigm for sustainable ecological design, i.e. microscopically self-organising a topological structure for energy and resource distribution within the system, thus optimising environmental performance by minimising the negative environmental impact on the natural environment. A holistic information system is designed for effective control and adaption of environmental performance of systems using the mechanisms of open systems evolution.

\section{References}

[1] Adami, C., What is complexity? BioEssays, vol. 24, pp 1085-1094, 2002.

[2] Bergós, J., Gaudí, the man and his work, Boston, Little, Brown and Company, 1999.

[3] Dmitry, K. C., The Main Principles of Simulation Modelling of the Sustainable Development Complexes System: Case of World Economy Complex Sciences, Lecture Notes of the Institute for Computer Sciences, Social Informatics and Telecommunications Engineering, Springer Berlin Heidelberg, 2009.

[4] Davies, P., Cosmic blueprint: new discoveries in nature's creative ability to order the universe, Philadelphia, Templeton Foundation Press, 2004.

[5] Emergence, Organisation and Dynamics of Living Systems, Santa Fe Institute, www.santafe.edu.

[6] Frazer, J., An Evolutionary Architecture (Themes VII). London, Architectural Association Publications, 1995.

[7] Ingersoll, R., Second Nature: On the Social Bond of Ecology and Architecture. Reconstructing Architecture: Critical Discourses and Social Practices. T. A. Dutton and L. H. Mann. Minneapolis, University of Minnesota Press, 1996.

[8] Jin, Y. L., Daoism, Nature and Man, 2005. 金岳霖, 道, 自然与人 生活·读书·新知三联书店. 
[9] Kauffman, S. A., The Origins of Order: Self-organisation and Selection in Evolution. New York, Oxford University Press, 1993.

[10] Kauffman, S. A., Investigations, New York, Oxford University Press, 2000.

[11] Kuhn, T. S., The Structure of Scientific Revolutions, Chicago, The University of Chicago Press, 1962.

[12] Leach, N., Swarm urbanism, Digital Cities, Architectural Design, 2009.

[13] Moore, S., Architecture, Esthetics, and Public Health. The Hand and the Soul: Ethics and Aesthetics in Architecture and Art. S. Illescu. Charlottesville, VA, University of Virginia Press, 2006.

[14] Nicolis, G. and I. Prigogine, Exploring complexity: an introduction. New York: W.H. Freeman, 1989.

[15] Prigogine, I., Introduction to thermodynamics of irreversible processes. New York, Interscience Publishers, 1961.

[16] Prigogine, I., The arrow of time. IN C.ROSSI \& E.TIEZZI (Eds.) Ecological Physical chemistry. Amsterdam, Elsevier, NL, 1991.

[17] Prokopenko, M., Boschetti, F. \& Ryan, A. J., An information-theoretic primer on complexity, self-organisation and emergence The 8th Understanding Complex Systems Conference, UIUC, 2007.

[18] Rogers, E. M., Physics for the inquiring mind; the methods, nature, and philosophy of physical science, Princeton, N.J., Princeton University Press, 1960.

[19] Shannon, C. E., The mathematical theory of communication, Urbana, University of Illinois Press, 1949. 